\title{
Regulation of Leaves Senescence by Virus-Induced Gene Silencing (VIGS) Modus Operandi in Arabidopsis
}

\author{
Allah Jurio Khaskheli ${ }^{1,2, *}$, Muhammad Ibrahim Khaskheli ${ }^{3}$, Muharam Ali ${ }^{2}$, Li Zhang ${ }^{1}$, \\ Asad Ali Khaskheli ${ }^{4}$ Hai Qing Liu ${ }^{1}$, Muhammad Azeem Khaskheli ${ }^{5}$, Syad Zakir \\ Hussain Shah $^{2}$ \\ ${ }^{1}$ Institute of Cell Biology, School of Life Sciences, Lanzhou University, Cheng Guan, Lanzhou-730000, Gansu, P.R. China; ${ }^{2}$ Department of \\ Biotechnology, Faculty of Crop Production, Sindh Agriculture University, Tandojam-70060, Pakistan; ${ }^{3}$ Department of Plant Protection, \\ Faculty of Crop Protection, Sindh Agriculture University Tandojam- 70060, Pakistan; ${ }^{4}$ Department of Animal Nutrition, Shaheed Benazir \\ Bhutto University of Veterinary \& Animal Sciences, Sakrand, Pakistan; ${ }^{5}$ Department of Plant Pathology, Sindh Agriculture University \\ Tandojam- 70060, Pakistan
}

Received: October 27, 2020; Revised: December 3, 2020; Accepted: December 5, 2020

\begin{abstract}
The final leaf developmental stage starts with nutrients salvage and ends at cells' death, whereby leaf yellowing is the first noticeable event during senescence. Yellowing of leaves starts at the margins and progresses to the interior of the leaves' blade. In this regard, there are only a few factors that are being demonstrated in involving the regulation of cell death by evaluating the leaf senescence appearances of knocking of mutants and identifying downstream target genes. Thus, the current research aimed to evaluate the efficiency of Virus-Induced Gene Silencing (VIGS) and its functional analysis for a potential regulation of leaves senescence in Arabidopsis. In the present study, the silencing of the plant by VIGS technique caused a narrative phenotype with a high level of transcript levels. Nevertheless, the phenotype is exemplified with a smaller size compared to the wild type (WT) with smaller roots, leaves, and overall plant bodies. Interestingly, the vector (VG)silenced plants showed intense yellowing of leaves developed at the bottom regions along with a smaller number of tillers from the base of the plants. Moreover, we also tested leaves of age-dependent silenced Arabidopsis plants and observed a reduction in size and number of leaf cells compared to that of non-silenced (WT) control plants. To understand the advanced regulatory molecular mechanisms, the efficiency of vector infection has been confirmed through changes that happened via the measurement of ion seepage and decreasing content of chlorophyll, measurement of SAG12, and PAGs gene expressions. In conclusion, VIGS approach play a critical role in leaves senescence.
\end{abstract}

Keywords: Arabidopsis, Chlorophyll, Senescence, Silencing, VIGS.

\section{Introduction}

Aging is a complex and highly regulated process involving the decay mechanism of photosynthesis, cessation of chloroplasts, and the degradation of biomolecules such as proteins, nucleic acids, and lipids (Al-Shomali et al., 2017). The first visible event during aging so far is that the leaves turn yellow (Quirino et al., 2000). The leaf senescence is a comprehensive response of leaf cells. It provides information about plant age, internal, and environmental indicators. Integrating the internal and external plant environment in given ecological zone help the plant in adapting aging process (Odiyi and Eniola, 2015).

There are various abiotic and biotic factors influencing the leaf senescence (Lim et al., 2007). However, knocking out genes is also important in the aging of any plant organ, such as fruits, flowers, and leaves (Chen et al., 2002; Guo et al., 2004). Therefore, in this study, we hypothesized and planned to observe the role of (VIGS-vectors) activity in the senescence of leaves. Given to that, Programmed Cells
Death is a positively active process involving the distinct expression of thousands of genes (Hee et al., 2010). Through genome-wide analysis of gene expression changes, several Arabidopsis genes encoding transcription factors can be identified (Harb et al., 2020). However, only a few factors are being demonstrated in involving the regulation of cell death by evaluating the leaf senescence appearances of knocking of mutants and by identifying downstream target genes (Balazadeh et al., 2008; Buchanan-Wollaston et al., 2005; Lin and Wu, 2004). In the present study, we focused on the manifold roles of $V G$ vector relationships in regulating the cells' death of plants. Nevertheless, the transcriptional factors family gene regulates cell death by different hormonal stress, environmental strain, and their role in retrograde signaling (Ryu et al., 2004). This emergent complexity needs to be discussed first to explore the commercialization of plants and understand the controlled molecular mechanism involved in it. Detailed studies of SAGs identities and its expression indicate that regulation of leaf senescence is a complex process (Orzaez et al., 2006); however, in the Arabidopsis, the age of even a single leaf plays an

\footnotetext{
* Corresponding author e-mail: aajkhaskheli2012@gmail.com.
} 
important role in discovering leaf durability (Liu et al., 2004; Lu et al., 2003).

In the present study, we investigated the VIGS method in Arabidopsis, which relates to leaf senescence. Further, we hypothesized that vectors (VG) either function only in plant leaves or in other organs and plant growth behaviors. Therefore, we assumed that the vector (VG) could be tested in the leaf senescence method compared to other organs of the Arabidopsis plant. Also, we have evaluated various aspects of the vector (VG) promoting leaf senescence and demonstrated that vector (VG) continues to promote leaf senescence.

\section{Materials and methods}

\subsection{Plant materials and growth circumstance}

The seeds of the Arabidopsis plant were sterilized first with $10 \%$ of Sodium Hypochlorite solution by proper shaking approximately for 20-30min. After that, under the Laminar Air Flow Cabinet, seeds were rinsed with sterilized distilled water about 8-10 times. Then seeds were grown on MS-basal medium under $4^{\circ} \mathrm{C}$ for 2-3 days and kept for two weeks at $25^{\circ} \mathrm{C}$. Seedlings were then transferred to soil media and infiltrated with vector solutions. The phenotypic observations have been made at two days interval of treatment (Guo et al., 2004).

\subsection{Vector construction and transformation}

Vector was constructed in advance. The two weeks old seedlings of wild type Arabidopsis were infected with vector (VG) and vector $(C)$ using vacuum infiltration method. Following infection, the seedlings were grown in soil media. After two weeks, seedlings were transferred to soil media. The samples were collected for DNA isolation to confirm the efficiency of vector transformation (Tanaka and Makino, 2009).

\subsection{Phenotypic examination yellowish discoloration}

Regular examinations were made every two days to characteristically check the yellowing of the leaves. However, in experiments with Arabidopsis mutant studies, leaf yellowing may have started at the tip, petiole side, and mid of the leaf (Hee et al., 2010).

\subsection{Ion leakage measurement}

The infected plants were collected at every week's interval after infiltrations. Collected leaves were boiled into hot water and then suspended solution was checked through the instruments. Further, the infected leaves of plants were then suspended into measuring solution and observed the ion leakage measurement by leakage activity through instruments as recommended by (Ryu et al., 2004).

\subsection{Measurement of chlorophyll content}

For measuring the leaves' senescence activity, measurement of chlorophyll content is very important. The content of chlorophyll was measured according to the method recommended by (Lu et al., 2003). The fresh plants materials were routed and chopped to small pieces. $0.5 \mathrm{~g}$ sample was measured through an analytical weight balance. The material was standardized by adding $100 \mathrm{ml}$ of $80 \%$ acetone. The mixture was homogenized, and the extract was separated. The extract was examined on the spectrophotometer for chlorophyll content.

\subsection{Extraction of total DNA and Polymerase Chain Reaction}

Arabidopsis affected leaves were subjected to total DNA extraction. Solution $2 \mathrm{X}$ CTAB $(10 \mathrm{ml})$ was preheated in a water bath at a temperature of $65-70^{\circ} \mathrm{C} .5 \mathrm{~g}$ of leaves was frozen in liquid nitrogen and, thereafter, the pulverized powder was transferred to a pre-heated solution $(50 \mathrm{ml})$. The test tube containing $10 \mathrm{ml}$ of the 2 XCTAB solution was centrifuged. Besides, CIA liquid was added, incubated at $37^{\circ} \mathrm{C}$ and shacked at $120 \mathrm{rpm}$ for 20 minutes. Slowly, precipitation buffer was added and shacked gently for 15-20 times until the DNA completely precipitated. Pre-cooled ethanol $\left(-20^{\circ} \mathrm{C}\right)$ was transferred and immersed in the following ethanol solution: $70 \%$ ethanol, 7 minutes, $100 \%$ ethanol, and 5 minutes. For PCR analysis, the kit manufacturer's procedure was followed. $1 \mu \mathrm{g}$ of total DNA was used. The specific primers used are listed in Table 1. The PCR reaction was performed for 30 minutes at $94^{\circ} \mathrm{C}$ for 5 minutes, the 30s at $94^{\circ} \mathrm{C}, 30$ s at $55^{\circ} \mathrm{C}$, and at $72^{\circ} \mathrm{C}$ for 30 minutes. The PCR products were then estranged on 1.0\% agarose gel. Alpha Ease FC-2200 software (Alpha Innotech, USA, version 3.2.1) was used to enumerate the absolute transcript values from the PCR.

\subsection{Statistical analysis}

Statistical analysis was performed on the obtained results according to the analysis of variance (ANOVA) technique. Treatments were compared using the least significant difference (LSD) at the $5 \%$ probability level. All calculations and statistical analyses were performed using the student software package 8.1.

\section{Results}

\subsection{VIGS plays a significant role in promoting the leaves senescence}

In our study, virus-induced gene silencing has been proved as an exceptional source of rapid advances in the field of genetics (Tripathi and Tuteja, 2007). The TRV can infect the different plant organs including carpopodia (Liu et al., 2002; Dinesh-Kumar et al., 2003), leaves (Fu et al., 2005), fruits (Lu et al., 2003), and roots (Orzaez et al., 2006) by agro-injection, agro-drench, agro-infiltration, and vacuum infiltration methods. In present study, we infected the Arabidopsis plant with investigated Vector (VG) efficiency in concern to senescence approaches by vacuum infiltrations method. The vacuum infiltration method was designed, whereby infected leaves were kept at $8^{\circ} \mathrm{C}$ for three days. Vector infected Arabidopsis leaves were subjected to phenotypic analysis every alternate day during the study period. Slightly, yellowish discoloration of leaves was seen at the start in all silenced leaves and nonsilenced leaves (Wild type). After 10 days of infiltration the vector $(V G)$ leaves were flattering to yellow faded instead of the vector $(C)$ (control) and wild type (Figure 5). For confirming the efficiency and effectiveness of vector $(V G)$, the changes occurred due to vector (VG) expression (Figure 3); ion leakage measurement; chlorophyll content, and SAG12 expression as the markers for senescence progression, were calculated. Significant difference (Figure 3) was noticed between vector (VG) and vector (c). Similarly, increased ionic leakage and SAG12 expression, decreased chlorophyll concentration were seen during 
senescence of leaves and the whole plants, which proved that vector (VG) played a critical role in leaves senescenc.

\subsection{Quantity of chlorophyll content highly reduced in vector (VG) infected plants}

Photosynthesis is a multi-stage process which plays a significant role in the growth and development of plant (Ayumi and Amane, 2009). In present study, we investigated chlorophyll concentrations in vector $(V G)$ and vector $(C)$ infected leaves of Arabidopsis. It has been significantly proved that vector (VG) had affected the concentration of chlorophyll content (Figure 6a). The chlorophyll concentration remarkably decreased in vector (VG) leaves of Arabidopsis (Figure 6a) compared to nonsilencing vector $(C)$, where chlorophyll concentration remained consistent. For further confirmation of these alternations, the expression of the PAG gene concerning to SAG12 gene was tested. The findings were confirmed by noticing slightly lower expression of PAGs and significantly higher expression of SAG12 in the vector (VG) (Figure 6c) compared to higher PAGs and lower SAG12 expression in the non-silenced vector $(C)$.

\subsection{VIGS implicating in the reduction of cells enlargement}

We further investigated that vector (VG) either affected the cell size enlargement or cell size reduction. We tested age-dependent leaves of the vector $(V G)$ silenced Arabidopsis plants. We categorized leaves as young leaves, mature leaves, partial senesced leaves, and fully senesced leaves. It was found that from beginning, when the leaves are becoming mature, had reduced size and as well as decreased the number of cells (Figure 7b) in vector (VG) leaves compared to that of non-silenced leaves vector (C) control. Regardless of Arabidopsis, the phenotype of plants is also differentiated with a persistently smaller size compared to that of non-silenced (vector C) and as well as wild type (WT). More interestingly, these features have given an image to the novelty of phenotype in the shape of more yellowing of leaves in silencing plants compared to the wild type (Figure 5). Further, we observed that vector (VG) suppressed to phenotype is responsible for the repression of cells and resulted in decreasing the size and structure of the overall plant body (Figure 7d). Therefore, vector (VG) was plausible as a strong sensitive transcription factor that was responsible for some key functions during the cellular behavior of plant structure.

\subsection{Method infiltrating by seed significantly affect the growth behaviors of Arabidopsis plant}

Further, we had investigated the silencing approach by the seed infiltration method in Arabidopsis. Only a few reports have been illustrated about the silencing approach by seed infiltration methods as well as young seedlings of Arabidopsis till to date. There are a few studies on silencing approaches by VIGS application to seedlings and seeds in the premature growing stage (Nagamatsu et al., 2007). It is very challenging to inoculate (VIGS) vectors and induce infection in very young seedlings and through seeds as well. Thus, we tried to inoculate the infection by these two novel infiltration methods in Arabidopsis and we found amazing results. For the seeds' infiltration method, we sterilized wild-type Arabidopsis seeds with $10 \%$ sodium hypochlorite for 20 min with continuous shakings. After that, sterilized seeds were infiltrated under vacuum infiltration with the solution containing vector $(C)$ and vector (VG) at equal OD of 2.0, and kept infected seeds for about 1 hour at $8^{\circ} \mathrm{C}$. After that, we grown the seeds on two different types of culture media containing MS-Basal supplemented with 25ng of specific antibiotics and MSbasal media. Silenced seeds grew well on both antibiotic supplemented and MS-basal media (Figure 2a). After the seeds were cultured on media, the plants were placed at $4^{\circ} \mathrm{C}$ for about three days and then kept at $25^{\circ} \mathrm{C}$ for further growth. The vector (VG) showed better germination rate at the supplementation of antibiotics (Figure 2a). Moreover, the length of roots and leaves were remarkably suppressed in vector (VG) plants compared to vector $(C)$ and wild type plants (Figure 4c, e). The phenotypes were measured after two weeks grown in the soil. The vector (VG) showed completely different phenotypes with a smaller size of whole plants, and the rate of growth was slightly slower compared to that of vector (C) and wild type (Figure 4d).

\section{Discussion}

It has been cited that VIGS is a valuable tool for functional analysis of genes in plants (Burch-Smith et al., 2004). The VIGS stimulated the knock-down/silencing of a particular gene expression by using a viral vector carrying a fragment of the target gene. Most of VIGS vectors were utilized for gene silencing in plant growth stages, such as leaves. On the other hand, several VIGS vectors were successfully induced in the reproductive organs, including flowers (Fu et al., 2005; Nagamatsu et al., 2007; Ayumi and Amane, 2009), and fruits (Lim et al., 2003; Andersson et al., 2004). In present study, we applied VIGS techniques through the infiltrating vector $(V G)$ for leaves senescence. The VIGS techniques was found better approach for testing functional analysis to the silencing of the vector $(V G)$ in the senescence of leaves. Moreover, the method proposed for the way to infiltration of Arabidopsis concluded that the entire aging process was comparable and provided a suitable experimental system. Thus, we also applied and followed the way with minor modification and used leaves, whole plants, whereby we found novel results.

\subsection{VIGS induced at early stages leaf senescence}

It is well understood that leaf senescence is a heritably controlled developmental process that ultimately leads to cell death. Obviously, under normal growth conditions, young leaves do not senesce. Perhaps, senescence inhibitors can effectively inhibit senescence during early leaf development and activate activators of the leaves age (Peitao et al., 2014). Interestingly, the role of the carrier (VG) was significantly different in Arabidopsis leaves. The overall observation of the silent leaves of Arabidopsis was analyzed, and it was shown that during the whole experiment, the vector (VG) and vector (C) and wild type leaves showed slightly yellow leaves at the beginning of infiltration. Later, the vector (VG) leaves turned yellow to fade, instead of the vector (C) and wild type. This view shows that the efficiency and effectiveness of the vector (VG) are more important. Although changes were assessed by measuring ion leakage activity, the expressions of chlorophyll, SAG12, and PRGs genes were used as markers of aging progress. We found that there are significant differences compared to the vector (VG) and 
the vector (C). Similarly, the increased ionic leakage and SAG12 expression contrasted with the decreased chlorophyll concentration observed during leaf senescence, and the whole plant vector (VG) also played a key role in leaf senescence. Like our findings few others reported that by transferring nutrients from senescent leaves and carefully adjusting leaf senescence to maximize the plant's adaptability, the differential expression of many genes should be used to precisely control its occurrence, progress, and completion. Recent applications of genomics technology have enabled the isolation of a class of genes, so-called senescence-associated genes (SAG), whose expression is increased in senescent leaves (Zentgraf et al., 2004; Fu et al., 2006).

\subsection{VIGS play a critical role in stimulating the instigation of leaf senescence}

The effects of the vector (VG) on leaf senescence were noted by comparison of the yellowish discoloration level of the vector (VG) with non-silent vector (C) and wild type during the age-related leaf senescence in Arabidopsis. On the $15^{\text {th }}$ day after infiltration, the leaves started to turn yellow in the vector (VG), but the vector (C) infected and wild-type leaves remained green (Figure 1a). The vector (VG) remained silent 25-30 days after infiltration, and those leaves turned completely yellow and revealed the signs of death. On the other hand, the vector (C) and wildtype leaves upheld their integrity and revealed only slight yellowish discoloration (Figure 5). For authenticity, leaf aging symptoms were further investigated, and typical senescence-related physiological markers were measured including photosynthesis-related gene expression, senescence-related genes expression (SAG12), and chlorophyll concentration (Figure 6b). After 3 weeks of infiltration, the chlorophyll content of the vector (C) and wild-type leaves began to decrease, while the silent leaves of the vector (VG) had lost $45-50 \%$ of chlorophyll content (Figure 6a). To be sure, similar findings have been reported concerning their acceleration, induction, and onset of leaf senescence, photosynthetic activity to reduce chlorophyll content in silenced plants (Al-Gabbiesh et al., 2015; Del et al., 2008). Besides, we reviewed that plant transcription factors of other gene families often have similar functions. For example, (NAC) and (WRKY) family genes are well-known aging-related transcription factors (Fu et al., 2006). More than 20\% of the 109 (NAC) family genes in Arabidopsis are specifically induced during development-triggered senescence (BuchananWollaston et al., 2003). Combining all these observations, the general findings suggest that the vector (VG) can play an amazing regulatory role in the initiation of leaf senescence, and as a transcription factor, it may control senescence by activating or inhibiting genes involved in the process by transcription.
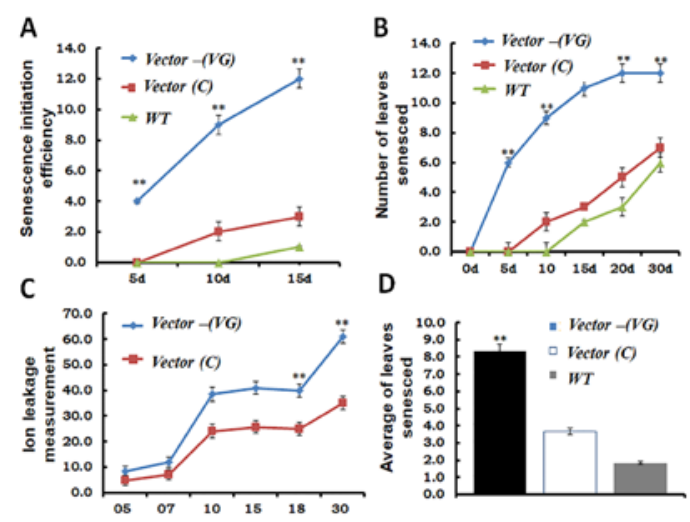

Figure 1. VIGS inducing leaves senescence. Senescence initiation efficiency (A), number of leaves senesced after infiltration (B), measuring of ion leakage after different time intervals (C), middling mean of overall senesced leaves of Arabidopsis (D). Asterisks denote statistically significant differences using student's $t$-test $(P<0.05, P<0.01)$. Error bar represent the $\mathrm{SD}$ of the average from three different biological replicates.
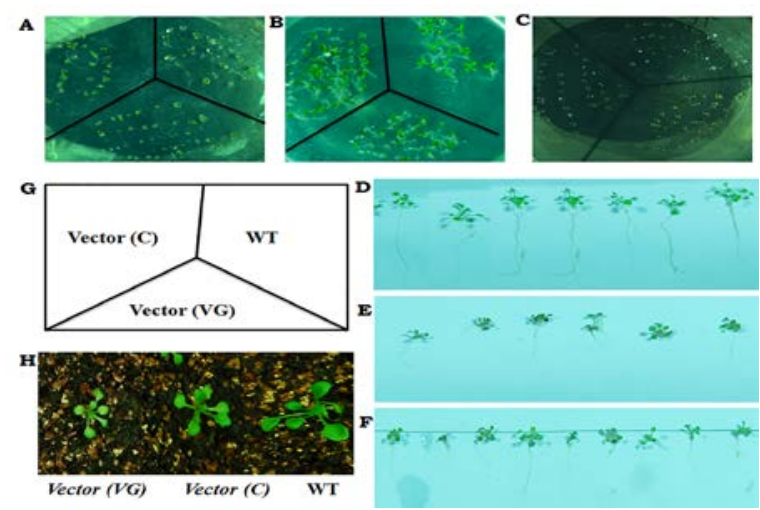

Figure 2. Virus Induced Gene Silencing by seed infiltration method. Germination percentage of vector $(V G)$, vector $(C)$ and WT at MS-basal media (A, B), Survival rate of vector (VG), vector $(C)$ and WT seeds in supplementation of antibody (C), Wild-type of two weeks old rooted seedlings (D), vector $(C)$ two weeks old rooted seedlings (E), vector (VG) two weeks old rooted seedlings (F), illustration of preparing plates (G), seedlings grow into soil and after 10 days vector (VG) showed remarkably low sized plant compare to that of wild type and vector $(C)$ and showed slight light color of leaves $\mathbf{( H )}$. Data are the average of three different biological replicates.
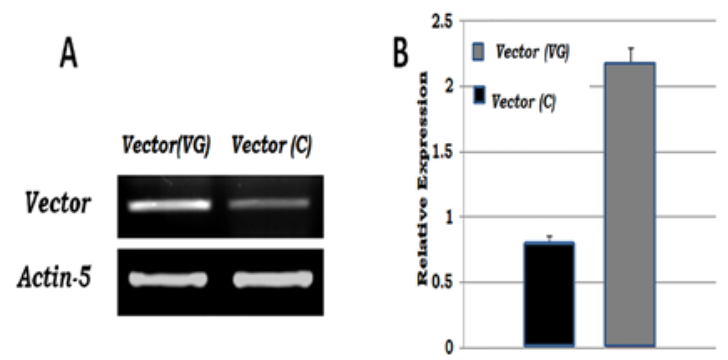

Figure 3. Relative expression level of vector (VG) affecting senescence in Arabidopsis. Qualitative expression measured by the PCR (A), PCR analysis of vector (VG) and vector (C) (B). Asterisks denote statistically significant differences using student's $t$-test $(P<0.05, P<0.01)$. Error bar represent the SD of the average from three different biological replicates. 

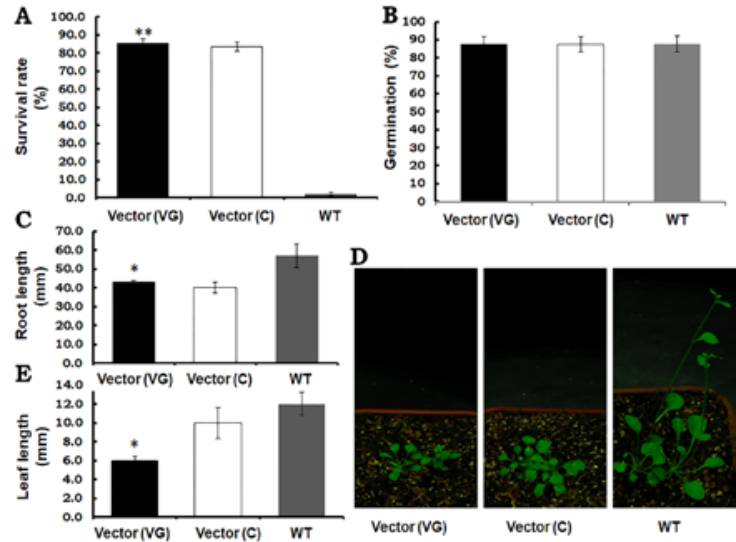

Figure 4. Effect of vector (VG) on growth behavior of Arabidopsis. Survival rate at supplementation media (A), Germination percentage at basal media (B), root length observed after germination (C), growth behaviors of Arabidopsis suppressed by vector (VG), vector (C) and wild type (D), leaves length measurement (E). Asterisks denote statistically significant differences using student's $t$-test $(P<0.05, P<0.01)$. Error bar represent the $\mathrm{SD}$ of the average from three different biological replicates.

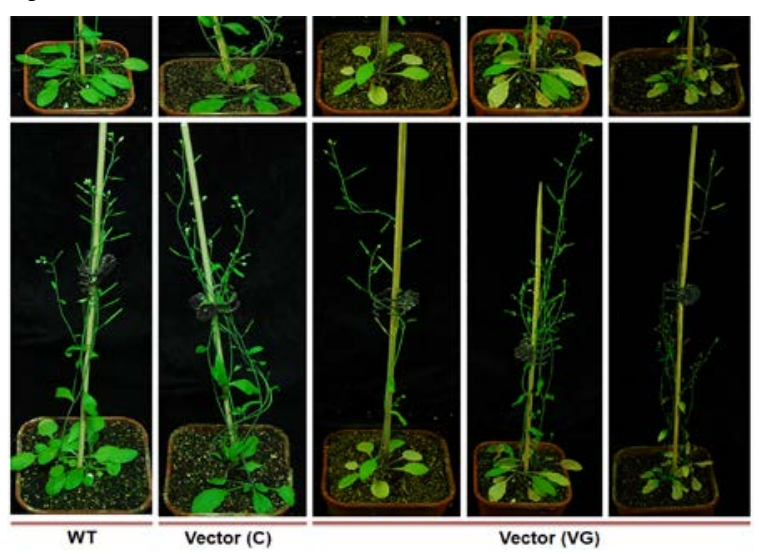

Figure 5. Phenotypic analysis of vector (VG) associated mutant lines of Arabidopsis. Data are the average of three different biological replicates.
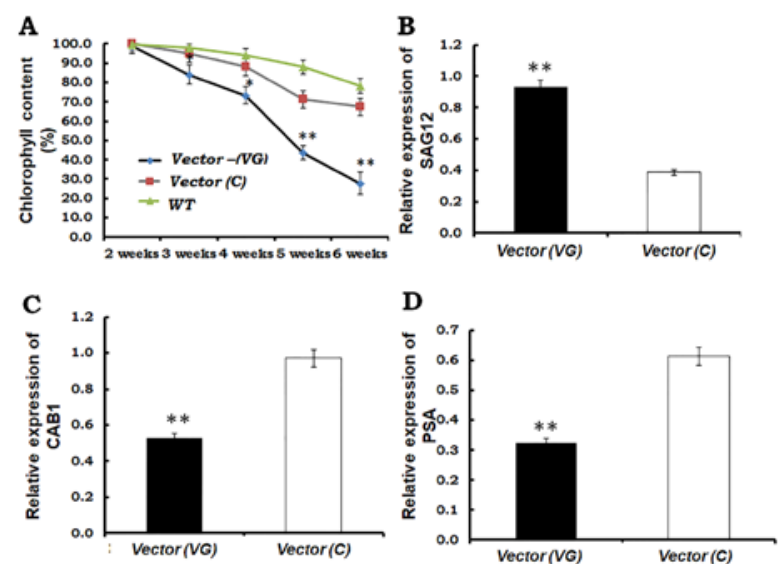

Figure 6. Expression pattern analysis with different marker genes and chlorophyll reduction in vector (VG) of Arabidopsis leaves. Chlorophyll content measurement (A), Relative expression analysis of SAG12 as senescence marker gene (B), Relative expression analysis of PSA as photosynthetic associated gene (C), Relative expression analysis of CAB1 as chlorophyll relating gene (D). Asterisks denote statistically significant differences using student's $t$-test $(P<0.05, P<0.01)$. Error bar represent the SD of the average from three different biological replicates.

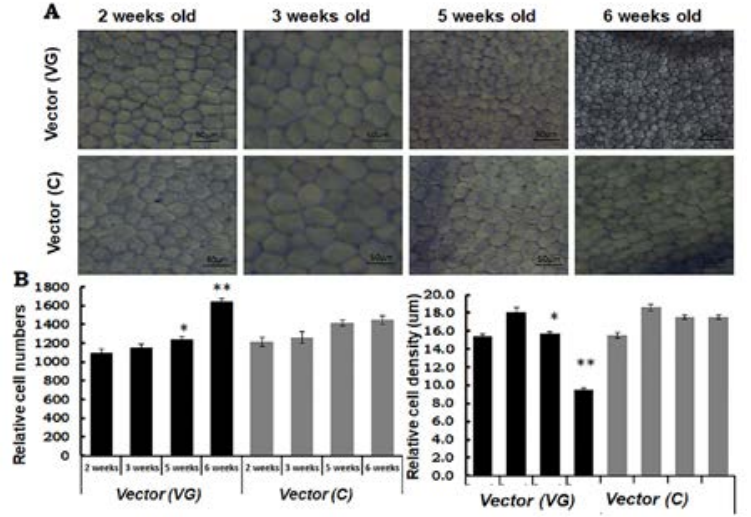

Figure 7. Effect of vector (VG) on cells sized reduction. Microscopic examination of leaves cells on different time intervals (A), Relative cells number and cell density observations (B). Asterisks denote statistically significant differences using student's $t$-test $(P<0.05, P<0.01)$. Error bar represent the SD of the average from three different biological replicates.

\section{Acknowledgment}

We would like to thank the School of Life Sciences, Lanzhou University, Gansu Province, P.R. China for providing research opportunity. We are also thankful to the Department of Biotechnology, Sindh Agriculture University, Tandojam, Pakistan which providing additional research resources for doing current research.

\section{Conflict of interest}

All authors declare that no conflict of interest exist.

\section{References}

Al-Shomali I, Sadder MT and Ateyyeha A. 2017. Culture Media Comparative Assessment of Common Fig (Ficus carica L.) and Carryover Effect. Jordan J Biol Sci., 10(1): 13-18.

Al-Gabbiesh A, Kleinwächter M and Selmar D. 2015. Influencing the contents of secondary metabolites in spice and medicinal plants by deliberately applying drought stress during their cultivation. Jordan J Biol Sci., 147(3379): 1-10.

Andersson A, Keskitalo J and Sjödin A. 2004. A transcriptional timetable of autumn senescence. Genome Bio., 5: 24-30.

Ayumi T and Amane M. 2009 Photosynthetic Research in Plant Science. P Cell Phys., 50: 681-683.

Balazadeh S, Rian DM, Pacho O and Mueller-Roeber B. 2008. Transcription factors regulating leaf senescence in Arabidopsis thaliana. $P$ Bio., 10: 63-75.

Buchanan-Wollaston V, Page T and Harrison E. 2005. Comparative transcriptome analysis reveals significant differences in gene expression and signaling pathways between developmental and dark/starvation-induced senescence in Arabidopsis. The P J., 42: 567-585.

Buchanan-Wollaston V, Earl S, Harrison E, Mathas E, Navabpour S, Page T and Pink T. 2003. The molecular analysis of leaf senescence: a genomics approach. P Biotech J., 1: 3-22.

Buchanan-Wollaston V, Page $\mathrm{T}$ and Harrison E. 2005. Comparative transcriptome analysis reveals significant differences in gene expression and signaling pathways between developmental and dark/starvation-induced senescence in Arabidopsis. The P J., 42: 567-585. 
Burch-Smith TM, Anderson JC, Martin GB and Dinesh-Kumar SP. 2004. Applications and advantages of virus-induced gene silencing for gene function studies in plants. The $P$ J., 39: 734746.

Chen JC, Jiang CZ, Gookin TE, Hunter DA, Clark DG and Reid MS. 2004. Chalcone synthase as a reporter in virus-induced gene silencing studies of flower senescence. $P$ Mol Bio., 55: 521-530.

Chen W, Provart NJ and Glazebrook J. 2002. Expression profile matrix of Arabidopsis transcription factor genes suggests their putative functions in response to environmental stresses. The $P$ Cell., 14: 559-574.

Del RA, del CR, López MG, Rivera-Bustamante RF and OchoaAlejo N. 2008. Virus-induced silencing of Comt, pAmt and Kas genes results in a reduction of capsaicinoid accumulation in chili pepper fruits. Planta., 227: 681-695.

Dinesh-Kumar SP, Anandalakshmi R, Marathe R, Schiff M and Liu Y. 2003. Virus-induced gene silencing. Methods Mol Bio., 236: $287-294$

Fu DQ, Zhu BZ, Zhu HL, Jiang WB and Luo YB. 2005. Virusinduced gene silencing in tomato fruit. $P$ J., 43: 299-308.

Fu DQ, Zhu BZ, Zhu HL, Zhang HX, Xie YH, Jiang WB, Zhao $\mathrm{XD}$ and Luo YB. 2006. Enhancement of virus-induced gene silencing in tomato by low temperature and low humidity. Mol Cells., 21: 153-160.

Guo Y, Cai Z and Gan S. 2004 Transcriptome of Arabidopsis leaf senescence. P Cell \& En., 27: 521-549.

Harb AM, AL-Hadid KJ and Sharab AS. 2020. Molecular and Biochemical Changes of Indole-3-Acetic Acid in the Expanding Leaves of Barley (Hordeum vulgare L.) under Salinity Stress. Jordan J Biol Sci., 13(1): 93-100

Hashem HA and El-Sherif NA. 2019. Exogenous Jasmonic Acid Induces Lead Stress Tolerance in Kidney Bean (Phaseolus vulgaris L.) by Changing Amino Acid Profile and Stimulating Antioxidant Defense System. Jordan J Biol Sci., 12(3): 345-353.

Hee K, Junyoung K, Jeongsik K, Ung L, In-Ja S, Jin HK, HyoYeon L, Hong GN and Pyung OL. 2010. The RAV1 transcription factor positively regulates leaf senescence in Arabidopsis. J Exp Bot., 14: 3947-3957.

Lim PO, Woo HR and Nam HG. 2003. Molecular genetics of leaf senescence in Arabidopsis. Trends P Sci., 8: 272-278.

Lim PO, Kim HJ and Nam HG. 2007. Leaf senescence. Ann Rev P Bio., 58: 115-136.

Lin JF and Wu SH. 2004. Molecular events in senescing Arabidopsis leaves. The P J., 39: 612-628.
Lin Z, Hong Y, Yin M, Li C, Zhang K and Grierson D. 2008. A tomato HD-Zip homeobox protein, LeHB-1, plays an important role in floral organogenesis and ripening. P J., 55: 301-310.

Liu Y, Nakayama N, Schiff M, Litt A, Irish VF and DineshKumar SP. 2004. Virus induced gene silencing of a DEFICIENS ortholog in Nicotiana benthamiana. P. Mol Bio., 54: 701-711.

Liu Y, Schiff M and Dinesh-Kumar SP. 2002a. Virus-induced gene silencing in tomato. $P$ J., 31: 777-786.

Lu R, Malcuit I, Moffett P, Ruiz MT, Peart J, Wu AJ, Rathjen JP, Bendahmane A, Day L and Baulcombe DC. 2003. High throughput virus-induced gene silencing implicates heat shock protein in plant disease resistance. EMBO J., 22: 5690-5699.

Lu R, Martin-Hernandez AM, Peart JR, Malcuit I and Baulcombe DC. 2003a. Virus-induced gene silencing in plants. Methods., 30: 296-303.

Nagamatsu A, Masuta C, Senda M, Matsuura H, Kasai A, Hong JS, Kitamura K, Abe J and Kanazawa A. 2007. Functional analysis of soybean genes involved in flavonoid biosynthesis by virus-induced gene silencing. P Biotech J., 5: 778-790.

Odiyi BO and Eniola AO. 2015. The Effect of simulated acid rain on plant growth component of Cowpea (Vigna unguiculata) L. Walps. Jordan J Biol Sci., 147(3379): 1-4.

Orzaez D, Mirabel S, Wieland WH and Granell A. 2006. Agroinjection of tomato fruits. A tool for rapid functional analysis of transgenes directly in fruit. $P$ Phys., 140: 3-11.

Peitao L, Changqing Z, Jitao L, Xiaowei L, Guimei J, Xinqiang J, Muhammad AK, Liangsheng W, Bo H and Junping G. 2014. RhHB1 mediates the antagonism of gibberellins to ABA and ethylene during rose (Rosa hybrida) petal senescence. The $P \mathrm{~J}$., 78: $578-590$

Quirino BF, Noh YS, Himelblau $E$ and Amasino RM. 2000. Molecular aspects of leaf senescence. Trends in P Sci., 5: 278-282.

Robertson D. 2004. VIGS vectors for gene silencing: many targets, many tools. Ann Rev P Bio., 55: 495-519.

Ryu CM, Anand A, Kang L and Mysore KS. 2004. Agrodrench: a novel and effective agroinoculation method for virus-induced gene. P Cell Rep., 31: 1713-1722.

Tanaka A and Makino A. 2009. Photosynthetic research in plant science. $P$ Cell Phys., 50(13): 681-683.

Tripathi S and Tuteja N. 2007. Integrated signaling in flower senescence: an overview. P Sig Behav., 2: 437-445.

Zentgraf U, Jobst J, Kolb D and Rentsch D. 2004. Senescencerelated gene expression profiles of rosette leaves of Arabidopsis thaliana: leaf age versus plant age. $P$ Bio., 6: 178-183. 